\title{
EVOLUTION OF A DEGENERATE COMPONENT IN A CLOSE BINARY SYSTEM
}

\author{
B.V. Frgma \\ Astronomical Council \\ USSR Academy of Sclences
}

Investigation of evolution of a degenerate component (helium or carbon-oxygen dwarf) in a close binary system is rather important. Such dwarfs may be candidates for nova or supernova stars. The first possibility has been studied by several authors (see review paper by Starrfield et al., 1976) and it has been shown that explosive hydrogen burning with enriched CNO abundance in the degenerate envelope of a massive carbon-oxygen dwarf may explain nova phenomena.

It is quite possible that during the flash the accreted envelope is not lost completely and in the course of accretion the core mass is increasing. The evolution of the central values of density and temperature is now the function of the mass accretion rate. Thus, formation of masaive helium or carbon-oxygen dwarfs is posaible in a close binary system. Special interest of this scenario is connected with explanation of supernova explosions in elliptical galaxies (Whelan and Iben, 1973; Gursky, 1976; Ergma and Tutukov; 1976; Ergma, 1976; Nomoto and Sugimoto, 1977; Ergme et al., 1978).

\section{A. Evolution of a Carbon-0xygen Dwarf.}

Ergma and Tutukor (1976) proposed a simple method for investigation of the evolution of central values of the density and the temperature of a c-0 dwarf. The mass accretion rate was taken equal to the mass rate through the hydrogen and hellum shell sources (gtationary ohell sources). It was shown that if $<10^{-7} \mathrm{M} / \mathrm{yr}$ carbon begins to burn when the central value of density is about $10 \% \mathrm{~g} / \mathrm{cm}^{2}$. According to hydrodynamic calculations (Chechetkin et al., 1978) for this case a neutron star forms after a supernova explosion.

Miyaji (1977) pointed out that for small accretion rates the evolution of central values of temperature and density takes place in the region where physical conditions change. If mass accretion rate is less than $10^{-8}$ 
$M \circ / y r$, central values of $T$ and $\rho$ may be in the region where $T<\theta$ and $\theta S r$, where $\theta$ is the Debye temperature, $\quad \Gamma$ is the Coulomb interaction energy over $k T$, and $\Gamma=120$. For this case matter is a quantum liquid. Besider the change of thermodymamical propert1es of matter, an important question arises connected with the value of the energy production rate. With decrease of temperature the energy generation rate in the $C^{12}+C^{12}$ reaction drastically decreases (E $\sim T^{30-20}$ ). Following Salpeter and Van Horn (1969) the strong screening factor

$$
\begin{aligned}
& H_{12}(0)=\ln \left[\frac{1+3.408 \rho_{s}^{1 / 3} / T_{9}+10.185 \rho_{9}^{1 / 3} / T_{9}^{26}}{\left(1+1.0374 \rho_{9}^{1 / 3} / T_{9}+1.8003 g_{9}^{1 / 3} / T_{9}^{2}\right)^{2}}\right]_{9}^{(1)} \\
& U_{s p}=84.175 / T_{g}^{1 / 3} \cdot\left(\beta-\frac{2.15 \beta^{3}+47.8 \beta^{9}}{1+17.1 \beta^{3}}\right) \\
& \beta=0.04494 \rho_{9}^{1 / 3} T_{8}^{2 / 3}, T_{9}=T / 40^{9}, \rho_{g}=\rho / 10(3)
\end{aligned}
$$

The energy generation rate $\mathcal{E}$ was taken after Fowler et al. (1975).

$$
\begin{aligned}
& \varepsilon_{0}=\varepsilon_{0} \exp \left(H_{12}(0)\right) \quad \operatorname{erg} / g / \mathrm{sec} \\
& \varepsilon_{0}=2.89 \cdot 10^{43} X_{12}^{2} \rho / T_{g}^{2 / 3} \exp \left(-84.165 / T_{9}^{1 / 3}\right)
\end{aligned}
$$

The pycnonuclear reaction rate was taken as (Hansen and Wheeler, 1969)

where

$$
\varepsilon_{\text {pye }}=2.67 \cdot 10^{63} \lambda^{7 / 4} \exp \left(-2.64 \lambda^{-1 / 2}\right) x_{12}^{2}(5)
$$

$$
\lambda=1.941 \cdot 10^{-7} \rho^{1 / 3}
$$

In Fig. 1 the values of $\mathcal{E}$ estimated by equations (4) and (5) with $\mathrm{X} j \mathrm{j}=1$ are given for various $\mathrm{T}$ and $\rho$. According to Fig. $f_{\text {for }} 1 \mathrm{gg}>10.0$ and $1 \mathrm{~g} T<7.9 \quad \varepsilon$ estimated by equation (4) Tapidly decreases and becomes less than epe (equation (5)). In their calculations Ergme and Tutukov (1976) used the strong screening factor by Salpeter and Van Horm (1969). According to their estimates for amall accretion rates $\leqslant 10^{-9} \mathrm{M} / \mathrm{yr}$ due to small energy production rate the central value of the density exceeds the density of oxygen neutronization $(I \mathrm{~g} S=10.24)$. Duncan et al. (1975) used the pycnonuclear reaction rate for $\mathcal{E}$ and concluded that in the course of accretion carbon burning in the pycnonuclear regime leads to explosive carbon burning. Regretfully our knowledge about the thermonuclear reaction rate and screening factor in the region of quantum Iiquid is rather poor. Due to 
this fact it is quite uncertain to say about neutronization of oxygen before carbon burning begins. On the other hand, for high values of central density $(I g \rho \approx 10.0)$ the uncertain convective transfer mechaniam which heats nearby layers, and may be of great importance for reaolving the problem of explosive carbon burning (Nomoto et al., 1976; Kudryashor et al., 1979), may be replaced by a more physical neutrino ignition mechanism (see Chechetkin et al., 1978). Besides, as it was shown by some authors, if $i_{8} \rho \gtrsim 9.95$ it is possible to form a neutron star after $\&$ supernova explosion.

B. Evolution of a Helium Dwerf.

The first rather approximate calculation of the evolution of an accreting helium dwarf was made by Ergma (1976). Nomoto and Sugimoto (1977) and Brgme et al. (1978) performed a full scale hydrostatic calculation of an accreting helium dwarf with the initial mass $\mathrm{M}=0.4 \mathrm{M}_{0}$. The results of calculations are presented in Table 1.

Table 1. Physical quantities at the helium ignition $\left(\varepsilon_{\text {Me }}=\varepsilon_{0}+\varepsilon_{\text {siF }}\right) M-$ mass of the helium core, $\dot{u}$ - accretion rate; the other quantities correspond to the ignition point.

\begin{tabular}{|c|c|c|c|c|c|c|}
\hline M(MO/Yr & M/MO & $\mathrm{Mr} / \mathrm{M}$ & IgP & $\lg T$ & $\psi$ & \\
\hline $1.6 \cdot 10^{-8}$ & 0.64 & 0.50 & 5.59 & 7.92 & 12 & Ergma et al. \\
\hline $1.6 \cdot 10^{-9}$ & 1.24 & 0 & 8.21 & 7.77 & 950 & Ergma et al. \\
\hline $4 \cdot 10^{-8}$ & 0.66 & 0.57 & 5.43 & 7.95 & 8.3 & $\begin{array}{l}(1978) \\
\text { Nomoto and }\end{array}$ \\
\hline $2 \cdot 10^{-8}$ & 0.78 & 0 & 6.86 & 7.87 & 67.3 & $\begin{array}{l}\text { Sugimoto (1977) } \\
\text { Nomoto and }\end{array}$ \\
\hline $1 \cdot 10^{-8}$ & 0.99 & 10 & 7.37 & 7.79 & 141 & $\begin{array}{l}\text { Sugimoto }(1977) \\
\text { Nomoto and } \\
\text { Sugimoto }(1977)\end{array}$ \\
\hline
\end{tabular}

According to our results $\left(\dot{\mu}=1.6 \cdot 10^{-8} \mathrm{Mo} / \mathrm{yr}\right.$ ) helium begins to burn in the outer shell. It is possible then that in the flash the outer layers would be mixed with layers of enhanced chemical composition (Ergma_et al., 1979). In the case of olow accretion (M $=1.6 \cdot 10^{-5} \mathrm{M} / \mathrm{gr}$ ) the subsequent helium burning in the core may lead to a supernova explosion (Mazurek, 1973; Nomoto and Sugimoto, 1977).

Thus, evolution of an acreting hellum or carbonoxygen dwarf in a close binary system may lead to formation of a messive dwarf. In such a dwarf burning of helium (or carbon) may lead to quite another scenario of explosion than thet of single stars. 


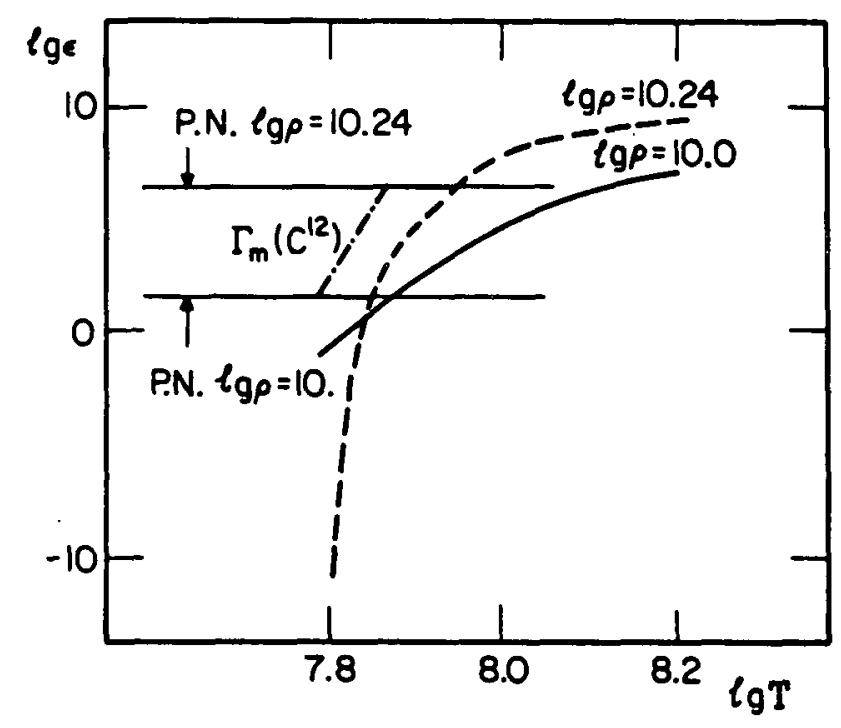

Fig. 1 - Values of $\mathrm{lg} \varepsilon$ for various $\mathrm{Ig} \mathrm{T}$ and $\mathrm{Ig} \rho$.

P.N. denote pycnonuclear rates for the indicated $1 \mathrm{~g} \rho$ from eq. (5); the dashed and solid curves are the strong screening rates from eq. (4); denotes the crystallization curve for $\mathrm{C}^{12}$.

\section{REPERENCES}

Chechetkin, V.M., Gershtein, S.S., Imshennik, V.S., Ivenova, I.N., Khlopov, M.Yu. 1978, Preprint IHEP, 78-158.

Duncan, J.J., Snell, R.I., Mazurek, T.J., Wheeler, J.C. 1975, Ap. Letters, 17, 19.

Ergma, E.V. 1976, Pisma Astron. Zh., 2, 544.

Ergma, E.V., Tutukov, A.V. 1976, Acta Astron., 26, 69.

Ergma, E.V., Rahunen, T., Vilbu, 0. 1978, Nauchnye Informatsil, 45, 124.

Ergma, E.V., Rahunen, T., Vilhu, 0. 1979, Astron. Zh. (in press).

Fowler, W.A., Cauglen, G.R., Zimmerman, B.A. 1975, Ann. Rev. Astrophys., 13, 69.

Hansen, C.J., Wheeler, J.C. 1969, Astrophys. Space Sci., 3. 464 .

Kudryashov, A.D., Shcherbatyuk, V.A., Ergma, E.V. 1979, Astrophys. Spece Sci. (in press).

Mazurek, T.J. 1973, Astrophys. Space Sci., 23, 365.

Miyaj1, S. 1977, private communication.

Nomoto, K., Sugimoto, D. 1977, PASJ, 29, 765.

Nomoto, K., Sugimoto, D., Neo, S. 1976, Astrophys. Space Sc1., 39, I 37.

Salpeter, E.E., Van Horn, H.M. 1969, Ap. J. $155,183$. Starrfield, S., Sparks, W.M., Truran, J.W. 1976, Proc. of IAU Symp. 73, p. 115.

Whelan, J., Iben, I.Jr. 1973, Ap. J., 186, 1007. 